\title{
Do minimalist shoes improve balance and foot strength in older adults?
}

\author{
Simon Franklin ${ }^{\mathrm{a}^{*}}$, Michael J. Grey ${ }^{\mathrm{b}}$ and François-Xavier $\mathrm{Li}^{\mathrm{a}}$ \\ ${ }^{a}$ University of Birmingham, Birmingham, UK \\ ${ }^{\mathrm{b}}$ University of East Anglia, Norwich, UK
}

\section{Introduction}

$30 \%$ of people over 65 years old and $50 \%$ of people over 80 years old have at least one fall per year (NICE, 2013). One avenue explored to attempt to reduce this fall risk is footwear.

Minimalist footwear is a way to simulate walking barefooted, offering no constriction to the foot allowing it to function naturally whilst providing a protective surface. Aside from allowing the foot to spread it is suggested, the thin flat sole and absence of restricting motion control structures increases the sensitivity of the sensory mechanisms and better activates the foot and lower leg muscles (Mckeon et al 2015).

Previous research has indicated both enhanced sensory feedback sensitivity (Machado et al 2016) and increased foot strength (Spink et al 2011) improve balance in older adults.

\section{Purpose of the study}

To investigate if wearing minimalist shoes in daily life for a 4 month period leads to improvements in balance and foot strength.

\section{Methods}

26 physically active adults (13 in intervention group, 4male, $71 \pm 4$ years, $25.13 \pm 4.36 \mathrm{BMI}$; 13 in control group, 3male, $71.8 \pm 3.7$ years, $23.09 \pm 3.43 \mathrm{BMI}$ ) volunteered.

All participants were assessed on static balance (postural sway; 3x30seconds, parallel stance, eyes closed), dynamic balance (functional reach test (FRT); 3xboth sides), gait (3x10m walk test, 3D motion capture), foot mobility magnitude and hallux strength (3xhallux plantar flexion MVIC on each foot) at baseline and after 4 months of wearing a minimalist shoe (Women's: Vivobarefoot ${ }^{\mathrm{TM}}$ Joy; Men's: Vivobarefoot ${ }^{\mathrm{TM}} \mathrm{RA}$ ) in daily life.

Footwear use (time spent wearing the minimalist shoes/day) and habitual activity (step count/day) were recorded for 1 week intervals within each month.

Qualitative data was collected prior to the start of the follow up session to evaluate their perceptions of the shoes and any changes felt in balance or confidence over the 4 month period.

Statistical comparisons were made using Paired Sample t-tests $(\mathrm{p}<.05)$.

\section{Results}

The following results are from the 13 participants in the intervention group. Participants showed a good level of activity with an average of $8360 \pm 4830$ steps per day and spent $414 \pm 151$ minutes per day wearing the minimalist shoes.

There was an increase in FRT performance from baseline $(30.45 \mathrm{~cm} \pm 6.36)$ to follow up $(33.98 \mathrm{~cm} \pm 4.00) ; \mathrm{t}(12)=2.43, \mathrm{p}=.032$ (Table1). This corresponded with increased strength in the left hallux from baseline $(37.54 \mathrm{~N} \pm 17.12)$ to follow up (48.74N \pm 24.00$) ; \mathrm{t}(12)=3.34, \mathrm{p}=.006$ (Figure1). 
There were no significant differences in postural sway, foot mobility, gait timing variability, gait speed, step length, step width or stance phase duration.

\begin{tabular}{|c|c|c|c|}
\hline & Right & Left & Average \\
\hline Baseline & 30.8 & 30.0 & 30.4 \\
& \pm 6.7 & $\pm 6.5^{*}$ & $\pm 6.4^{*}$ \\
\hline Follow & 34.1 & 33.8 & 34.0 \\
Up & \pm 4.1 & $\pm 4.5^{*}$ & $\pm 4.0^{*}$ \\
\hline
\end{tabular}

Table1. Functional Reach Test performance $(\mathrm{cm})$ at baseline and follow up (means \pm s.d.). $*=$ sig. diff. from baseline to follow up $(\mathrm{p}<.05)$.

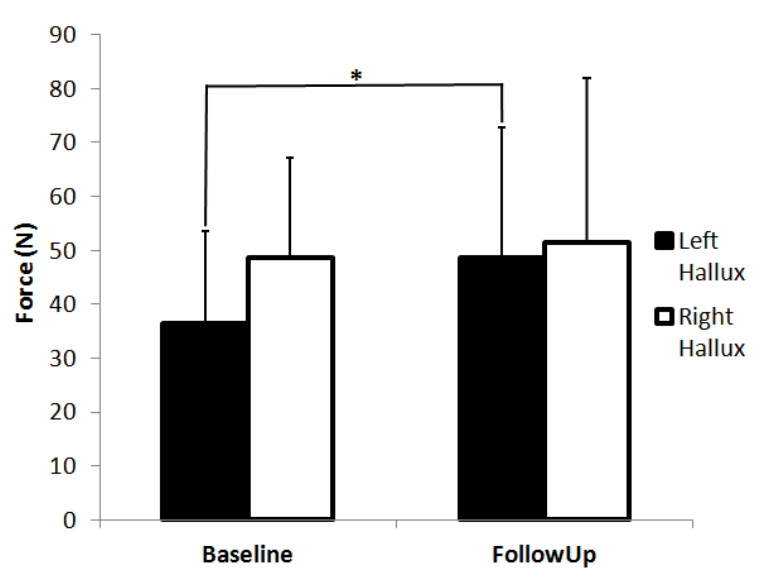

Figure1. Hallux plantarflexor maximum strength at baseline and follow up (means with error bars depicting s.d.) $*=$ sig. diff. from baseline to follow up $(\mathrm{p}<.05)$.

12 out of the 13 participants reported improved confidence in their balance with 8 of those feeling their balance had improved since wearing the minimalist shoes and the remainder reporting no change.
Reasons they gave for the improvement was greater awareness of the surface as well as improved weight/pressure distribution across the foot due to the flat and wide shoe.

\section{Discussion and conclusion}

This study demonstrated daily use of minimalist footwear could have a beneficial effect on the balance of older adults. Increased hallux strength and reach ability further suggests walking in minimalist footwear activates the foot muscles to a greater extent, thus leading to improvements in foot strength and consequently balance.

Whilst we observed no improvements in postural sway or the gait measures associated with poor balance control, the qualitative data suggest that outside of a laboratory environment improvements have been witnessed in both confidence and balance itself.

The participants in this study were habitually very active with good baseline performance thus greater improvements may be seen in the less active amongst this population.

The intervention group will be compared to the control group upon completion of data collection.

\section{References}

Falls: NICE clinical guideline 161; (2013) Mckeon, P.O. et al (2015) Br J Sports Med 49, 290

Machado, A.S. et al (2017) Rev Bras Reumatol 57(1), 30-36

Spink, M.J. et al (2011). Arch Phys Med Rehabil 92, 68-75 